\title{
A case report of duplex-guided femoral angioplasty in a patient with severe renal insufficiency still an option to consider
}

\begin{abstract}
Introduction: One of the main concern of percutaneous transluminal angioplasty (PTA) is the risk of renal injury in nephropathic patients. A valid alternative is represented by $\mathrm{CO}_{2}$ angiography, which is however contraindicated in patients with chronic obstructive pulmonary disease and is not always available in hospitals. In these cases, the role of duplex guided balloon angioplasty (Du-PTA) should be reassessed.
\end{abstract}

Case presentation: We describe the case of a 75years-old nephropathic Caucasian male patient who underwent a duplex-guided percutaneous transluminal angioplasty (Du-PTA) for a $10-\mathrm{cm}$ occlusion of his right femoral superficial artery causing rest pain. A review of the literature about the use of duplex ultrasound for the endovascular treatment of infrainguinal vascular disease in selected high-risk nephropathic patients was also performed.

Conclusion: In well selected patients who present with critical limb ischemia and are at high risk for contrast-induced nephropathy, and when $\mathrm{CO}_{2}$ angiography cannot be performed, Du-PTA of femoral-popliteal district can still be a safe and effective alternative to conventional PTA and should be taken into account.

Keywords: duplex guided angioplasty, critical limb ischemia, renal failure, case report, diabetology, angiography
Volume 5 Issue 4 - 2016

\author{
Daniela Mazzaccaro,' Davide Foresti,' Maria \\ Teresa Occhiuto,' Silvia Stegher,' Giovanni \\ Malacrida,' Erika Defebis, ${ }^{2}$ Paolo Righini, \\ Giovanni Nano' \\ 'First Unit of Vascular Surgery, IRCCS Policlinico San Donato, \\ Italy \\ ${ }^{2}$ University of Milan, Graduate School of Vascular Surgery, Italy
}

Correspondence: Daniela Mazzaccaro, IRCCS Policlinico San Donato, First Unit of Vascular Surgery Piazza Edmondo Malan I, 20097 San Donato Milanese MI, Italy, Tel +39025277434I, Email danymazzak83@libero.it

Received: October 31, 2016 | Published: December 19, 2016

\section{Abbreviations}

PTA, percutaneous transluminal angioplasty; Du-PTA, duplexguided percutaneous transluminal angioplasty; CLI, critical limb ischemia; DSA, digital subtraction angiography; COPD, chronic obstructive pulmonary disease; SFA, superficial femoral artery; PSV, peak systolic velocity; CIN, contrast induced nephropathy; eGFR, estimated glomerular filtration rate

\section{Introduction}

Since the concept of bypass surgery using vein graft was introduced by Kunlin ${ }^{1}$ in 1949, Vascular Surgeon's approach to steno-obstructive pathology of lower limbs has significantly changed. The cornerstone was placed in 1960, when Dotter et al., ${ }^{2}$ introduced the concept of endovascular revascularization with percutaneous transluminal angioplasty (PTA). The importance of this acquisition is evidenced by the growing space given to the endovascular approach as the first choice in many cases of infrainguinal arterial disease causing critical limb ischemia (CLI). The Consensus Document of the Italian Society of Diabetology together with the Italian Society of Radiology and the Italian Society of Vascular and Endovascular Surgery published in $2014^{3}$ underlined the role of PTA as a first line therapy, most of all in patient with diabetic lower limb ulcerations.

However one of the main concerns of this procedure is the risk of renal injury in these same patients, who often are affected by preexistent diabetic nephropathy. In these patients, in fact, even a small amount of iodinate contrast medium can precipitate a precarious balance of the renal function, eventually leading to the need for dialysis treatment in the short to medium term.
For this reason Cluley et al., ${ }^{4}$ in 1991 suggested the use of Duplex-guided Percutaneous Transluminal Angioplasty (Du-PTA) for the treatment of femoral-popliteal disease in patient with critical limb ischemia or severe disabling claudication and concomitant risk factors such as creatinine serum levels $>1.5 \mathrm{mg} / \mathrm{dL}$. Another valid alternative for patients with chronic kidney disease or with a known allergy to contrast medium, is represented by $\mathrm{CO}_{2}$ angiography, whose efficiency and effectiveness has been demonstrated in the literature to be comparable to that of conventional Digital Subtraction Angiography (DSA). ${ }^{5}$ However, the $\mathrm{CO}_{2}$ angiography is relatively contraindicated in patients with chronic obstructive pulmonary disease (COPD). Moreover, it requires a special delivery system to prevent air contamination and gas compression, which is not always available in all hospitals. We describe the case of a 75years-old patient affected by COPD and diabetic nephropathy, who was successfully treated with Du-PTA for a $10-\mathrm{cm}$ occlusion of his right femoral superficial artery causing CLI with rest pain. A review of the literature was also performed about the treatment of femoral-popliteal disease using DuPTA in selected high risk patients.

\section{Case presentation}

A 75-year-old Caucasian male with CLI on his right leg was admitted at our Institute fifteen months ago. He had a clinical history of COPD at GOLD C class, renal failure (preoperative serum creatinine $4.22 \mathrm{mg} / \mathrm{dL}$ ) and type 2 diabetes mellitus treated with insulin. Seven and six years before he had undergone endarterectomy and stenting of his right and left internal carotid artery, respectively. He was also affected by coronary artery disease which was previously treated with a coronary artery bypass graft and afterwards with implantation 
of drug eluting stent on circumflex artery for recurrent angina. The patient had been complained for right leg rest pain for 1month without ischemic ulcers or gangrene. Ultrasonography on his right leg showed the presence of a $10-\mathrm{cm}$ occlusion at the mid third of superficial femoral artery (SFA) (Figure 1), with a poor distal flow which was recorded from the popliteal to the pedal artery. Both posterior tibial and peroneal arteries were occluded. On the left leg, both femoral and popliteal pulses were palpable. Transcutaneous oximetry on the first toe was $35 \mathrm{mmHg}$ on the right side and $58 \mathrm{mmHg}$ on the left side in a sitting position. The precise location of the occlusion after ultrasound mapping was marked preoperatively with a sign on the skin to facilitate the treatment in the operating room.

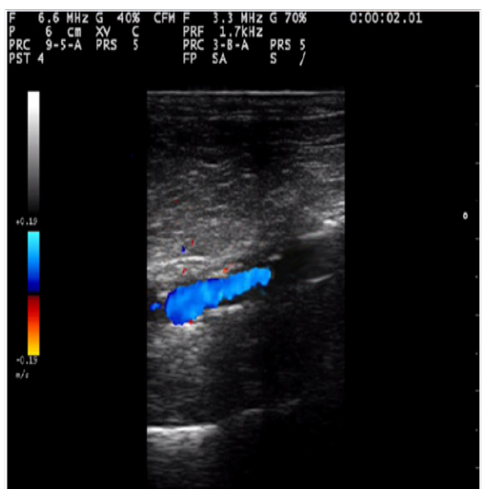

Figure I Preoperative color image of the lesion.

A local anesthesia (1\% lidocaine) was performed at the right groin. A $7.5 \mathrm{MHz}$ probe of a Esaote Mylab 50-X Vision allowed the correct visualization of the common femoral artery for an ipsilateral antegrade percutaneous approach with a $5 \mathrm{Fr}$ sheath. A bolus of 2500IU of unfractionated heparin was administered intraarterially to have an activated clotting time of more than 200seconds, then an hydrophilic. 035 inch guide wire with $\mathrm{J}$ tip (Terumo) and a directional $5 \mathrm{Fr}$ angle tapered directional catheter (Berenstein) were successfully advanced throughout the occlusion (Figure 2) and towards the infrapopliteal segment in the correct lumen, under the direct echographic visualization. The diameter of the SFA at the target lesion was measured and a $4 \times 80 \mathrm{~mm}$ over-the-wire balloon (Evercross, Covidien) was chosen for dilatation. Duplex control showed the complete resolution of the occlusion from the morphological point of view, without any sign of dissection or recoil and with normalization of both the peak systolic velocity (PSV) and the PSV ratio. Moreover, a direct bi-phasic flow was recorded on the pedal artery. No stent was needed. Postoperative course was uneventful and the patient was discharged on $2^{\text {nd }}$ postoperative day on acetyl salicylic acid $100 \mathrm{mg} /$ daily. At 1-yearfollow-up the patient was still asymptomatic and a duplex ultrasound showed neither recurrent nor residual stenosis.

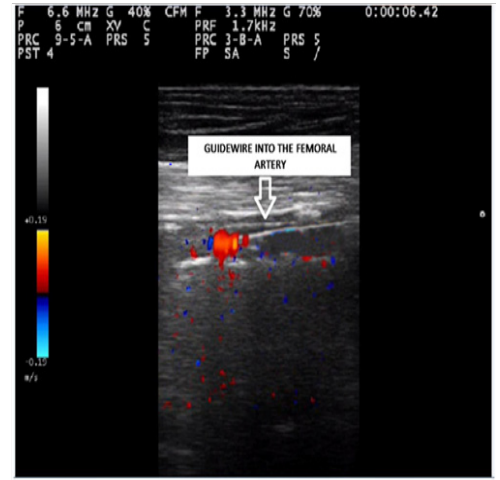

Figure 2The hydrophilic 035 inches guide wire with J tip (Terumo) advanced throughout the occlusion in the correct lumen.

\section{Literature review}

A systematic review searching the MEDLINE, Scopus, Web of Science, ClinicalTrials.gov and Cochrane Central Register of Controlled Trials (CENTRAL) databases was also conducted using the key-words "duplex-guided angioplasty". Nine papers published between 1996 and 2015 were analyzed for the review (Table 1), as they specifically addressed the clinical use of ultrasound as a substitute of fluoroscopy in the endovascular treatment of infrainguinal disease. Papers reporting cumulative results after duplex guided angioplasty of both suprainguinal and infrainguinal vascular disease was excluded. A total of 603 arteries $^{6-14}$ (490 femoral-popliteal, 80 infrapopliteal, 33 failing infrainguinal bypass grafts) were treated using duplexguided angioplasty with a reported technical success of $84.6 \%-97 \%$. Procedural complications occurred in up to $12.5 \%$ of reported cases, being mainly not related to the use of ultrasound. In all examined cases, duplex-guided balloon angioplasty was reported to be a safe and effective technique that allowed renal patients to experience limb salvage and relief from claudication without the risk of developing dye-induced acute renal failure. Other reported advantages included direct visualization of the puncture site, accurate selection of the proper size of balloon and stent, confirmation of the adequacy of the technique also by hemodynamic parameters, and avoidance of any radiation, both for the patient and for the operators.

Table I Resume of literature review

\begin{tabular}{|c|c|c|c|c|c|c|}
\hline Author & Year & Arteries & $\begin{array}{l}\text { Technical } \\
\text { success }\end{array}$ & $\begin{array}{l}\text { Procedural } \\
\text { complication }\end{array}$ & $\begin{array}{l}\text { Primary } \\
\text { patency }\end{array}$ & $\begin{array}{l}\text { Limb } \\
\text { survival }\end{array}$ \\
\hline \multirow[t]{2}{*}{ Ascher ${ }^{6-11}$} & $\begin{array}{l}2005- \\
2008\end{array}$ & 360 SFA-pop & $95 \%$ & n.r. & TASC A: $90 \%$ & n.r. \\
\hline & & 80 infra-pop & $96 \%$ & & TASC B: $59 \%$ & \\
\hline
\end{tabular}

TASC C: $52 \%$

TASC D: $46 \%$

(at 12 mths)

$\begin{array}{lllllll}\text { Marks }^{12} & 2006 & 33 \text { failing infrainguinal by pass grafts } & 97 \% & 6 \% & 69 \% \text { at } 6 \text { mths } & 100 \% \text { at } 6\end{array}$


Table Continued.

$\begin{array}{lllllll}\text { Ahmadi }^{13} & 2002 & \text { 104 SFA-pop } & 84.60 \% & 12.50 \% & \begin{array}{l}60.2 \% \text { at } 6 \\ \text { mths }\end{array} & \text { n.r. } \\ \text { Katzenschlager }^{14} & 1996 & 26 \text { SFA-pop } & 96.10 \% & \text { n.r. } & \begin{array}{l}80.9 \% \text { at } 6 \\ \text { mths }\end{array} & \text { n.r. }\end{array}$

SFA, superficial femoral artery; Pop, popliteal artery; n.r., not reported; mths, months

\section{Discussion}

Contrast-induced nephropathy (CIN) is a clinical condition in which an impairment of the renal function (an increase in serum creatinine by more than $25 \%$ or $44 \mu \mathrm{mol} / \mathrm{l}$ ) occurs within 3 days following the intravascular administration of a iodinate contrast medium in the absence of an alternative etiology. ${ }^{15}$ The pathogenesis of CIN has not been fully elucidated yet, however association between volume of administrated contrast media and the incidence of CIN has been well demonstrated by SeeligerandColl15, who reported a risk of CIN which doubles with every $20 \mathrm{ml}$ of contrast administered.

Patients with pre-operative renal insufficiency with creatinine serum level $>1.5 \mathrm{mg} / \mathrm{dL}$ and/or diabetes mellitus have been demonstrated to be at high risk of CIN. ${ }^{16}$ Unfortunately, these same subjects most frequently are affected by peripheral vascular disease causing critical lower limb ischemia. Their comorbidities however may contribute to the choice of an endovascular, less invasive approach rather than open surgery for the treatment of their vascular disease. CIN may be prevented16by preoperative volume expansion with isotonic saline infusion and sodium bicarbonate $0.84 \%$, associated with $\mathrm{N}$-Acetyl cysteine administration which has a proven antioxidant and vasodilator effects. ${ }^{17}$ Nevertheless, in some cases the risk to develop CIN remains high even if the best medical therapy is performed. In 1991 Cluley et al., ${ }^{4}$ suggested the use of Du-PTA for the treatment of femoral-popliteal disease in patient with critical limb ischemia or severe disabling claudication and concomitant risk factors such as creatinine serum levels $>1.5 \mathrm{mg} / \mathrm{dL}$. Since then, few cases have been reported in the literature using this technique, and the reason is probably why $\mathrm{CO}_{2}$ angiography ${ }^{5}$ has been introduced too as a valid alternative to conventional DSA, being as safe and effective as DSA, maybe superior to du-PTA and avoiding the risk of any contrast media. However, the $\mathrm{CO}_{2}$ angiography is relatively contraindicated in patients with COPD. Moreover, it requires a special delivery system to prevent air contamination and gas compression, which is not always available in all hospitals. When $\mathrm{CO}_{2}$ angiography cannot performed, Du-PTA must be taken into account as a valid alternative to conventional DSA. In our Institution, we don't have the special delivery system for $\mathrm{CO}_{2}$ angiography. Moreover, the patient was affected by COPD which relatively contraindicated the use of $\mathrm{CO}_{2}$, so duplex-guided angioplasty was the preferred choice. The most representative case-series of Du-PTA have been reported by Asher et al. ${ }^{6-11}$ who treated 360 cases of occlusive and stenotic lesions of the femoral-popliteal segment and 80 cases at the infrapopliteal segment from September 2003 to June 2005 with du-PTA, with an overall technical success rate of $95 \%$ and $96 \%$ respectively. Stents were also placed when needed, without any problems 8 . The same Authors ${ }^{12}$ published in 2006 a series of 25 patients treated with du-PTA for primary assisted patency of failing infrainguinal femoral-popliteal by passes, with a technical success in $97 \%$ of the cases. Limb salvage and primary patency rate at 6 month were $100 \%$ and $69 \%$ respectively.

Du-PTA has been reported also for the treatment of iliac lesions in highly selected cases. In 2010 Kawarada $^{18}$ reported two cases of du-PTA in the iliac artery in patients with chronic renal insufficiency at high risk for developing CIN. Krasznai et al., ${ }^{19}$ resumed that experience in a series of 35 patients with iliac lesions and an estimated Glomerular Filtration Rate (eGFR) $<60 \mathrm{ml} / \mathrm{min} / 1.73 \mathrm{~m}^{2}$ or allergy for contrast media. Treatment of iliac lesions however may be hampered by the difficult visualization of the lesions with the ultrasound in a hostile abdomen. The advantage of the echo guided intervention is the real time monitoring of the procedure, from the correct location of the antegrade arterial puncture to the guide wire progression toward the central lumen and possibly in the intraluminal way. Balloon diameter and length can also be chosen according to the arterial measurements obtained by duplex guidance. Moreover, the immediate postoperative results can be assessed not only by morphology but also from the hemodynamic point of view. On the other side, the depth of the localization may hamper visualization of the superficial femoral artery at the adductor hiatus. Moreover, highly calcified plaques may also affect the correct visualization of the lesion.

\section{Conclusion}

In well selected patients who present with critical limb ischemia and are at high risk for $\mathrm{CIN}$ and when $\mathrm{CO}_{2}$ angiography cannot be performed, Du-PTA of femoral-popliteal district can still be a safe and effective alternative to conventional PTA and should be taken into account. Du-PTA has the advantage of direct visualization of the percutaneous access site, accurate selection of the proper size of the devices balloon and stent and confirmation of the efficacy of the technique by both hemodynamic and morphologic parameters, without the risk of iodinate contrast material exposure.

\section{Consent}

Written informed consent was obtained from the patient for publication of this case report and any accompanying images. A copy of the written consent is available for review by the Editor-in-Chief of this journal.

\section{Authors' contribution}

Each Author has contributed substantially to the research, preparation and production of the paper and approves of its submission to the Journal.

\section{Acknowledgements}

None.

\section{Conflict of interest}

The author declares no conflict of interest.

\section{References}

1. Kunlin J. The treatment of arterial obstruction by vein graft. Arch Mal Coeur. 1949;42:371.

2. Dotter CT, Judkins MP. Transluminal treatment of arteriosclerotic obstruction. Description of a new technic and a preliminary report of its application. Circulation. 1964;30:654-670. 
3. Aiello A, Anichini R, Brocco E, et al. Treatment of peripheral artery disease in diabetes :A consensus of the Italian Societies of Diabetes (SID) Radiology (SIRM) and Vascular and Endovascular Surgery (SICVE). Nutr Metab Cardiovasc Dis. 2014;24(4):355-369.

4. Cluley SR, Brener BJ, Hollier LH, et al. Ultrasound guided balloon angioplasty is a new technique for vascular surgeons. Am J Surg. 1991;162(2):117-121.

5. Cho KJ. Carbon dioxide angiography: scientific principles and practice. Vasc Specialist Int. 2015;31(3):67-80.

6. Ascher E, Hingorani AP, Marks N. Duplex-guided balloon angioplasty of lower extremity arteries. Perspect Vasc Surg Endovasc Ther 2007;19(1):23-31.

7. Ascher E, Marks NA, Hingorani AP. Duplex-guided infrainguinal balloon angioplasty and stenting. A 4-year experience. J Cardiovasc Surg. 2008;49(2):151-158.

8. Ascher E, Marks N, Hingorani A, et al. Duplex guided endovascular treatment for occlusive and stenotic lesions of the femoro popliteal arterial segment:a comparative study in the first 253 cases. $J$ VascSurg. 2006;44(6):1230-1238.

9. Ascher E, Hingorani AP, Marks N, et al. Predictive factors of femoropopliteal patency after suboptimal duplex-guided balloon angioplasty and stenting:is recoil a bad sign? Vascular. 2008;16(5):263-268.

10. Ascher E, Marks NA, Schutzer RW, et al. Duplex-guided balloon angioplasty and stenting for femoropopliteal arterial occlusive disease:an alternative in patients with renal insufficiency. J Vasc Surg. 2005;42(6):11081113.

11. Ascher E, Marks NA, Hingorani AP, et al. Duplex-guided balloon angioplasty and subintimal dissection of infrapopliteal arteries:early results with a new approach to avoid radiation exposure and contrast material. JVascSurg. 2005;42(6):1114-1121.
12. Marks NA, Hingorani AP, Ascher E. Duplex Guided Balloon Angioplasty of failing infrainguinal bypass graft. Eur J Vasc Surg Endovasc Surg. 2006;32(2):176-181.

13. Ahmadi R, Ugurluoglu A, Schillinger M, et al. Duplex ultrasound-guided femoropopliteal angioplasty: initial and 12-month results from a case controlled study. J Endovasc Ther. 2002;9(6):873-81.

14. Katzenschlager R, Ahmadi A, Minar E, et al. Femoropopliteal artery:initial and 6-month results of color duplex US-guided percutaneous transluminal angioplasty. Radiology. 1996;199(2):331-334.

15. Seelinger E, Sendeski M, Rihal CS, et al. Contrast-induced kidney injury:meccanism, risk factors and prevention. Eur Heart J. 2012;33(16):2007-2015.

16. Golshahi J, Nasri H, Gharipour M. Contrast-induced nephropaty: a literature review. $J$ Nephropathology. 2014;3(2):51-56.

17. Tepel M, Van Der Giet M, Schwarzfeld C, et al. Prevention of radiographic contrast agent induced reductions in renal function by acetilcysteine. $N$ Engl J Med. 2000;343(3):180-184.

18. Kawarada O, Yoki Y, Takemoto K. Practical use of duplex echoguided recanalization of chronic total occlusion in the iliac artery. $J$ VascSurg. 2010;52(2):475-478

19. Kasznai AG, Sigterman TA, Welten RJ, et al. Duplex-guided percutaneous transluminal angioplasty in iliac artery occlusive disease. Eur J Vasc Endovasc Surg. 2013;46(5):583-587. 